\title{
ZigBee- based Wireless Electronic Scale and its Network Performance Analysis for the Application of Smart Billing System in Super Markets
}

\author{
R. Sivakumar' ${ }^{1}$ A. Maheswari ${ }^{2}$ and P. Pushpa ${ }^{2 *}$ \\ 'Department of Electrical and Electronics Engineering, Periyar Maniammai University, \\ Thanjavur-613403, India; sivan.nit@gmail.com \\ 25chool of Computing, SASTRA University, Thanjavur 613401, India; maheswariamudha@gmail.com, \\ pushpapadmanaban3@gmail.com
}

\begin{abstract}
There is the greatest demand for electronic weighing scale in vegetable shops, health industries and especially to measure the tiny things. This paper proposes a Wireless electronic weighing scale designed using ultra low power MSP430 controller and with ZigBee wireless communication module. The weighing scale measures the weight with the help of strain sensor and the processor, which has an inbuilt FRAM memory and Real time clock and calendar. This makes the scale to update the weighing databases more accurately and instruction with host PC in a wireless manner. The ZigBee cluster tree network is formed to communicate with more than one electronic scale and the network performance is analyzed using NetSim. The Visual Basic language is developed for user interface in PC side for generating bills.
\end{abstract}

Keywords: MSP430FR5739 Microprocessor, NetSim, PPSEN-10245 Strain Sensor, ZigBee Module

\section{Introduction}

Electronic scale has a wide range of application in commercial and industrial area. It is the upcoming trend to be extensively used in many weighing applications. It will provide accurate and reliable output compare to the conventional weighing scale. The major advantage of electronic weighing scale is accuracy, precision and reliability. Owing to its advantages, electronic scale finds more popularity in vegetable shops, health industries and especially to identify the measurement of the tiny things. The prominent features of the system are low cost, easy maintenance, flexible design and multipurpose.

The electronic weighing scale available is in price computing scale mode along with printer. The major drawback of the existed scale is it requires a dedicated PC. So the paper proposed an approach to overcome this problem.
Thereby, all the weighing details and database of the consumer can be stored in Host PC through wireless communication. The ZigBee is considered for wireless communication. The major advantage of this system is the change in commodity price can be updated automatically from host PC. More than one wireless electronic scale can be connected to the Host PC by the implementation of ZigBee cluster tree network. With all this the weighing database from more than one weighing scale can be transferred to Host PC and the bills of the purchase can be collected from Host PC itself. This will reduce the long queues and provide a sophisticated shopping at vegetable and retail shops.

\subsection{Existed System}

The significant drawback in traditional scale is its inability of storing detailed transactions information. It requires 
manual operation to store time, date and weight of goods. Also, the change in commodity price can be updated only through manual operators. It makes the process so complicated to use and difficult to get sales report and goods consumption habit of consumers. It is also hard to have better inventory management on goods. To overcome the difficulties the large supermarket uses expensive electronic scale but it did not have the capacity to store the information and it is not affordable for ordinary users. Later, an electronic weighing scale is developed with the features like price computing operation along with the printer. But the drawback is it requires a dedicated PC to store all the detailed transaction information for analyzing the consumer habits and also hard to enter the weight manually.

\subsection{Proposed System}

This paper introduces a wireless electronic scale based on ultra low power MSP430 microprocessor and ZigBee wireless module. It is capable of downloading the commodity information wirelessly from personal computer and store sales data, detailed transaction information to host PC and so on. The main prominent feature of this scale is that it acts like master- slave concept. By using ZigBee cluster tree network more than one weighing scale can be attached and the weighing database can be transferred to host $\mathrm{pc}$, thereby it avoids the use of dedicated PC for each weighing scale ${ }^{13}$.

\section{System Architecture}

The system comprises a MSP430FR5739 microcontroller module, ZigBee wireless communication module, Strain sensor detection module and host PC as shown in Figure 1.

\subsection{MSP430FR5739}

MSP430FR5739 is of ultra low-power microcontroller is the nucleus of the system ${ }^{1}$. Load sensor's output signal is initially conditioned by the use of inverting amplifier and instrument amplifier. Then it is processed through an ADC module for analog to digital conversion which is inbuilt in MSP430FR5739. The main advantage of MSP430FR5739 has embedded FRAM nonvolatile memory. It has 10-bit ADC, five 16-bit timers, and more. The main features are Universal Memory $=$ Program + Data + Storage, RealTime Clock (RTC) With Calendar and Alarm Functions and Power Management System. The advanced features make the application more reliable ${ }^{12}$.

\subsection{FRAM Memory}

MSP430FR5739 stores the memory relevant information and process the AD result in FRAM. FRAM is low power and can be used as non-volatile memory instead of Flash. RAM does not need a special sequence to write data nor does it require a higher programming voltage. A microcontroller with integrated FRAM is used to store both data and code and can be written quickly and provides practically limitless write durability.

\subsection{Real-time Clock/Calendar}

The Real-Time Clock module offers configurable clock counters and it is inbuilt in nature. It includes seconds, minutes, hours and calendar mode provides day of week, month, and year and with leap year correction. In this processor the calendar mode is supported only by RTC_B. It has interrupt capability, Selectable BCD or binary format, Programmable alarms, Calibration logic for time offset correction and Operation in LPMx.5.

\subsection{Strain Sensor Unit}

The system includes type PPSEN-10245 strain sensor. The maximum load capacity of strain sensor is approximately around $50 \mathrm{~kg}$. The major advantage of this strain sensor is it firms the signal of sensor with finest $1 \mathrm{~g}$ resolution and aggregate of 10,000 counts. Since, the stain sensor output voltage is very minimum, it requires amplification before $\mathrm{AD}$ conversions. The one-order low-pass filter and Instrument amplifier forms the conditioning circuit. The OPA333 series of CMOS operational

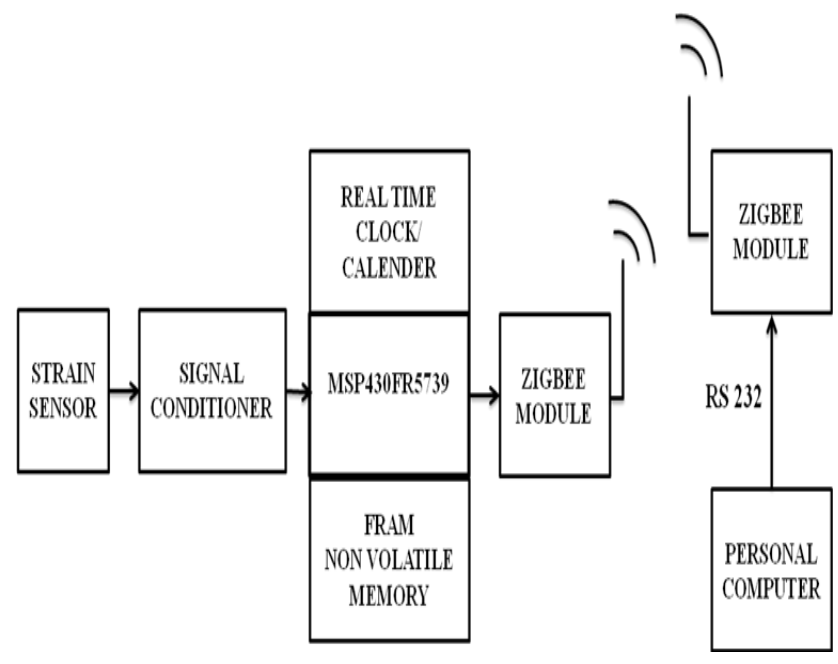

Figure 1. System Architecture. 
amplifiers provide temperature, near-zero drift over time and a minimum offset voltage $(10 \mu \mathrm{V}$ max). It offers current amplifiers with low quiescent, high-precision and $100 \mathrm{mV}$ high-impedance inputs in common-mode range beyond the rails, and within $50 \mathrm{mV}$ rail-to-rail output. Supply range is from $+1.8 \mathrm{~V}( \pm 0.9 \mathrm{~V})$ to $+5.5 \mathrm{~V}$ $( \pm 2.75 \mathrm{~V})$.

\subsection{Amplification Unit}

The AD624instrumentation amplifier is a low noise, high gain accuracy, high precision, high linearity and temperature coefficient with low gain. It is developed ideally for use with load cells, strain gauges, and low level transducers. It is primarily used for the application that needs high resolution in the field of data acquisition systems. The AD624C provides an output and input offset voltage drift of less than $10 \mu \mathrm{V} /{ }^{\circ} \mathrm{C}$ and $0.25 \mu \mathrm{V} /{ }^{\circ} \mathrm{C}$. The $80 \mathrm{~dB} \mathrm{CMRR}$ at unity gain $(130 \mathrm{~dB}$ at $\mathrm{G}=500)$ and at $\mathrm{G}=1$ the maximum nonlinearity is $0.001 \%$.

\subsection{Wireless Communication Unit}

Wireless Communication Module comprises ZigBee. Each weighing scale is connected to ZigBee modules for wireless transmission of weighing database and another ZigBee module to the host PC. The ZigBee technology provides long battery life, flexible and easy installation, low data rate(less than $250 \mathrm{Kbps}$ ) and integrated intelligence for network set-up and message routing. The ZigBee operates in $2.4 \mathrm{GHz}$ band and the operational range is $(10-75 \mathrm{~m})$. The maximum child and power is 254 and $1 \mathrm{~mW}$. The main function of ZigBee is to transfer the weighing database in a wireless manner to host PC.

\subsection{Host PC Unit}

Bills are generated in host PC using Visual Basic Language. ZigBee module and printer is connected to a $\mathrm{PC}$ to receive the weighing database from weighing scales. The database from more than one weighing scales are collected without collision by forming a cluster tree network with beacon enabled mode and Acknowledgement request. The commodity prices are updated to weighing scale from a PC. So, all the purchased bills from different weighing scales are collected at the host PC itself. This will reduce the long queues in the billing unit and provide a good ambience in large supermarkets.

\section{Description of ZigBee Wireless Communication System}

\subsection{ZigBee Characteristics}

ZigBee Alliance along with IEEE 802.15.4 has developed a unique standard based wireless technology named ZigBee. It is designed to address the unique needs of low power, low cost, extreme security and to support a maximum number of 255 web node operations. The transmission rate is low and it has a network structure of Master/Slave attributes with two-way communication. Under idle condition ZigBee is kept in sleep mode and activation time required for conversion between work and sleep is very less around $15 \mathrm{~ms}$, making ZigBee fairly power saving ${ }^{10}$. The Physical and MAC layer is based on IEEE 802.15.4 and the upper layers specifications are described by ZigBee protocol stack. The system's data transmission reliability is increased by collision prevention mechanism used in $\mathrm{ZigBee}^{2}$. Based on transmission power the module is designed considering the transmission distance between the nodes. Although the blocks in the building of super markets reduce the range of communication, using ZigBee cluster tree network and making some nodes as router can significantly overcome the issues of transmission in different horizontal and vertical floor with longer distance $^{3}$. But, as for the effect of noise interference it uses the Carrier Sense Multiple Access-Collision Avoidance (CSMA/CA) channel access mechanism, transmission power control and dynamic frequency selection to avoid channel collision ${ }^{4-6}$.

\subsection{ZigBee Cluster Tree Network Formation}

The issues of transmission while using multiple weighing scales connected with ZigBee module can be effectively overcome by forming Cluster Tree Network. So the cluster-tree network has been formed between the number of weighing scales and all the weighing scales transmit its weighing database to host PC and commodity prices from host PC in a two way communication like Master/Slave ${ }^{11}$.

In a mesh network the distinct case is the cluster-tree topology with one routing path between pair nodes and with a beacon-enabled mode in the distributed synchronization mechanism. The duty-cycle is managed dynamically in a per-cluster basis and worst-case network dimensioning is also possible to take out, in terms of end-to-end delays for worst-case message and buffer occupation. However, low duty-cycles of 100 percent down to 0.006 percent are only 
available in beacon-enabled mode ${ }^{7}$. This makes the system more reliable and synchronization for wireless transmission of data by forming cluster tree network with beaconenabled mode. Figure 2 shows the Cluster Tree Network formation of multiple weighing scales with host PC. The simulation output of Cluster Tree Network formation and its performance is described in section 5 . The Figure 2 comprises of nine nodes representing nine weighing scales forming a Cluster Tree network, where all the nodes act as FFD, such that it performs the functions of FFD.

\section{ZigBee Wireless Network Performance}

\subsection{Creating Network Scenario}

Tetcos in association with Indian Institute of Science developed a stochastic discrete event NetSim Simulator. Computer Aided Network Design and Analysis Research Environment provide tools, software libraries and algorithms for developing NETSIM.

The network models and restoration algorithms are facilitated by CANDARE ${ }^{8}$. The network performance of ZigBee is analyzed using NETSIM standard version simulation tool by creating the scenarios. The network scenario has been created by using nine ZigBee nodes and with one PAN coordinator. A typical network topology is arranged in a cluster tree format with parent-child relationships between nine ZigBee nodes and one PAN coordinator as shown in Figure 3. The properties such as datalink layer, physical layer, channel frequency of Zigbee PAN coordinator are represented in Table 1.

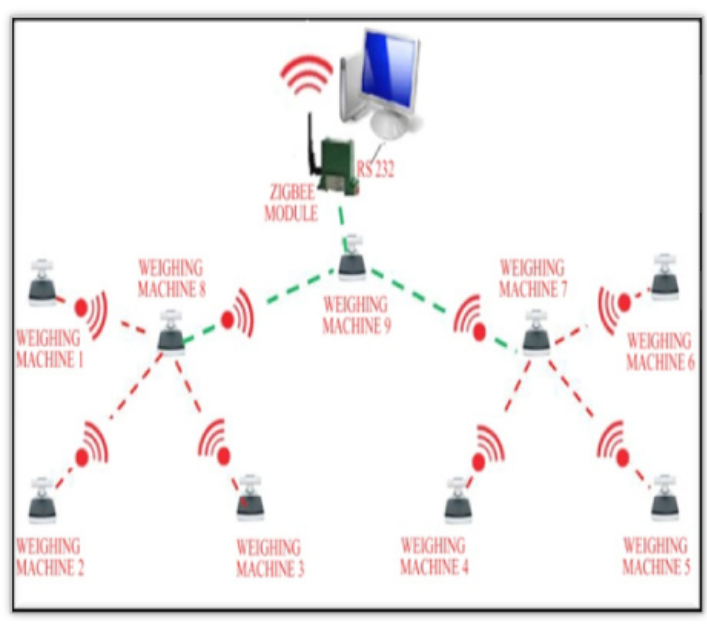

Figure 2. Wireless Communication Model.

\subsection{First Scenario Setup}

The first scenario has been created for the application of Wireless electronic Scale for Smart Billing System with nine nodes within a distance of $100 \mathrm{~m}$ range. The nodes form a Cluster Tree Network and each node properties are described in Table 2.

\subsection{Second Scenario Setup}

Similarly, the second scenario has been made with nine nodes within a distance of $100 \mathrm{~m}$ range. The nodes form a Cluster Tree Network and each node properties are described in Table 3.

\section{Simulation Results}

Here, a set of simulation is performed to explore the performance of ZigBee under realistic scenario. The simulation results concerns user level throughput, simulation time, payload delivered, Frames generated, transmitted, received and collided, CCA counts, successful and failed CCA and routing overhead across nine number of nodes and in two different scenarios are analyzed.

\subsection{Network Statistics}

\subsubsection{First Scenario}

The ZigBee network simulation uses Unslotted CSMA/ $\mathrm{CA}$ and it results in packet transmission after a random back off, CCA. Then it is followed by ACK packet and turn-around time and the specific time for each of it is set by the IEEE 802.15.4 standard.

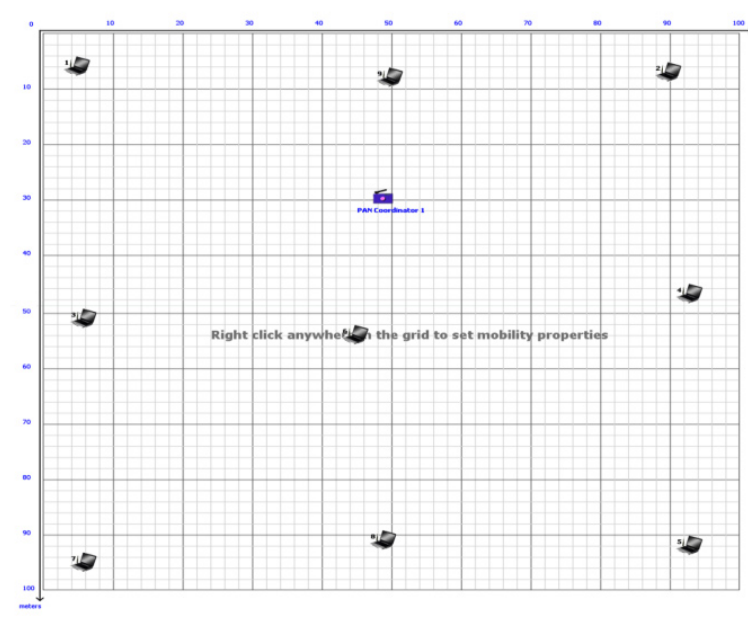

Figure 3. Node Setup Scenario using NetSim. 
ZigBee- based Wireless Electronic Scale and its Network Performance Analysis for the Application of Smart Billing System in Super Markets

Table 1. ZigBee PAN coordinator and Environment Properties

\begin{tabular}{ll}
\hline Properties & Pan Coordinator \\
\hline Data Link Layer & \\
Protocol & IEEE 802.15 .4 \\
Beacon Mode & Enabled \\
Beacon Order & 15 \\
Super frame Order & 15 \\
GTS Mode & Enabled \\
Super frame Duration & $15.36 \mathrm{~ms}$ \\
Battery Life Extension & True-1 \\
Maximum Back off Exponent & 5 \\
Minimum Back off Exponent & 5 \\
Max Frame Retries & 3 \\
MAX CSMA Back off & 4 \\
Unit Back off Period & 20 Symbols \\
Min CAP Length & 440 Symbols \\
GTS descriptor Persistent Time & 4 secs \\
Physical Layer & \\
Protocol & IEEE 802.15 .4 \\
Frequency Band & 2.4 GHz \\
Data Rate & $250 \mathrm{Kbits} / \mathrm{s}$ \\
Chip Rate & $2000 \mathrm{McPs}$ \\
Symbol Rate & $62.5 \mathrm{KSymbolsPS}$ \\
Modulation Technique & OQPSK \\
Min LIFS Period & 40 Symbols \\
Min SIFS Period & 20 Symbols \\
Unit Back off time & 20 Symbols \\
Turn Around Time & 12 Symbols \\
Phy SHR Duration & 3 \\
Phy Symbol Per Octet & 0.4 \\
CCM Mode & Carrier Sense Only \\
Receiver Sensitivity & $-85 \mathrm{dbm}$ \\
ED Threshold & $-95 \mathrm{dbm}$ \\
Properties & \\
Channels & Nonment \\
Frequency & Phoss \\
Channel Characteristics & \\
\hline
\end{tabular}

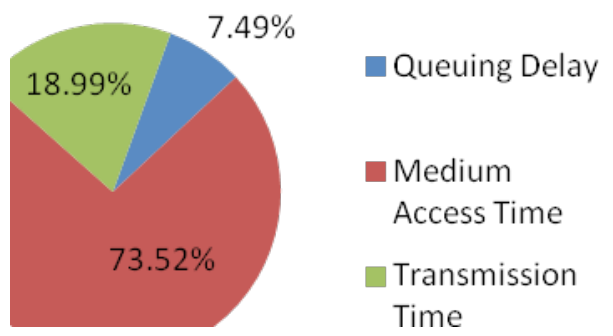

Figure 4. Network Performance Delay Report of First Scenario.
Table 2. Properties of First Scenario Nodes

\begin{tabular}{ll}
\hline Node Properties & Node (1-9) Range \\
\hline Transmission & Point to Point \\
Destination & As per Cluster Tr \\
Traffic Type & Data \\
Service Type & CBR -Constant Bit Rate \\
Mean Packet Size & $10-100$ bytes \\
Mean Inter Arrival Time & $1000-5000 \mathrm{~ms}$ \\
Data Link Layer & \\
Protocol & IEEE 802.15 .4 \\
Device Type & FFD \\
Retry Limit & 1 \\
Ack request & Enabled \\
Physical Layer & \\
Protocol & IEEE 802.15 .4 \\
Transmitter Power $(\mathrm{mW})$ & $0-50 \mathrm{~mW}$ \\
Transmitter Range & $100 \mathrm{~m}$ \\
\hline
\end{tabular}

Table 3. Propertiesof Second Scenario Nodes

\begin{tabular}{ll}
\hline Node Properties & Node (1-9) Range \\
\hline Transmission & Point to Point \\
Destination & As per Cluster Tr \\
Traffic Type & Data \\
Service Type & CBR -Constant Bit Rate \\
Mean Packet Size & $5000-10000$ bytes \\
Mean Inter Arrival Time & $15000-20000 \mathrm{~ms}$ \\
Data Link Layer & \\
Protocol & IEEE 802.15 .4 \\
Device Type & FFD \\
Retry Limit & 7 \\
Ack request & Enabled \\
Physical Layer & \\
Protocol & IEEE 802.15 .4 \\
Transmitter Power $(\mathrm{mW})$ & $0-50 \mathrm{~mW}$ \\
Transmitter Range & $100 \mathrm{~m}$ \\
\hline
\end{tabular}

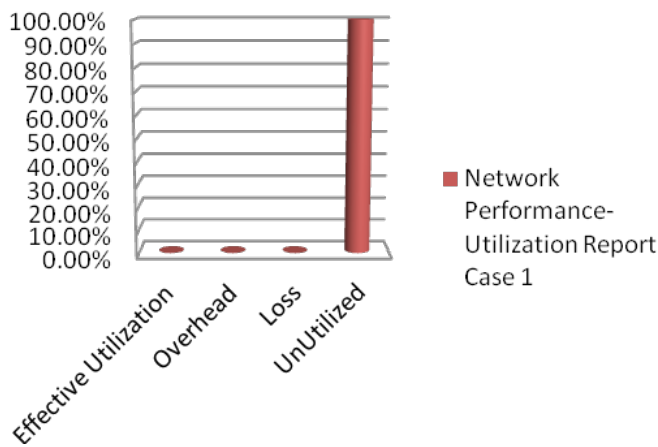

Figure 5. Network Performance Utilization Report of First Scenario. 
First scenario network statistics is described in Table 4. As per the node properties in this scenario, the range of Mean Packet size used is 10-100 bytes with beacon enabled and ACK request. The User level throughput results in $0.025 \mathrm{kbps}$ with the payload delivery of 280 bytes. CCA report a busy medium using detection of a signal compliant with same spreading characteristics and modulation of PHY that is presently in use by the device. Of the CCA counts of 2644 the successful count is 2644 with 0 counts of failed CCA. Routing overhead bytes of this scenario is 53076 bytes.

\subsubsection{Second Scenario}

Similarly, second scenario has been formed with the range of Mean Packet size 5000 to 10000 bytes with beacon enabled and ACK request. The User level throughput results in $7.592 \mathrm{kbps}$ with the payload delivery of 894580 bytes. Of the CCA counts of 61350 the successful count is 61334 with 15 counts of failed CCA. Routing overhead bytes of this scenario is 1238205 bytes. The scenario network statistics is described in Table 5.

\subsection{Network Performance Analysis}

The delay report is one of the main criteria in evaluating the network performance. The delay report majorly consists of Queuing Delay, Medium Access Time and Transmission Time respectively. The NetSim simulator keeps on tracking of the Queuing delay received by the packet transmitter. The difference of time between the time the packet gets submitted for transmission to the time when it successfully completes transmission is called Queuing delay.

This metric also includes the duration waiting time for channel access of the packet it was queued at the station. Thereby it illuminates the delay characteristics of the protocol being simulated. The user uses this quantity for network

Table 4. Network Statistics- First Scenario

\begin{tabular}{lr}
\hline \multicolumn{1}{c}{ Metrics } & Value \\
\hline User Level Throughput (kbps) & 0.025 \\
Simulation Time (ms) & 10000 \\
Payload delivered (Bytes) & 280 \\
Frames Generated & 71019 \\
Frames Transmitted & 2637 \\
Frames Received & 7 \\
Frames Errored/Collided & 21 \\
CCA counts & 2644 \\
Successful CCA & 2644 \\
Failed CCA & 0 \\
Routing Overhead (Bytes) & 53076 \\
\hline
\end{tabular}

Vol 7 (3) | March 2014 | www.indjst.org response more than the network oriented metric of overall inter arrival time. Whereas, the Medium Access Delay is defined as the packets average back off delay at MAC layer before it is dropped after several failed retransmission or successfully transmitted in an interval. Due to the increase in value of MAD there is a chance of occurrence of either or two possibilities. First of this is that the channel seems to be busier so that the node has to hold over for a long time to have a transmission opportunity. Secondly there is an increase in the number of retransmission because of higher level of collision. After each failed transmission the node returns to back off stage. Therefore medium access delay indicates both the collision and medium busyness around the node. Transmission time refers to the time of successful transmitted packet in an interval.

\subsubsection{First Scenario}

The network performance delay report and utilization report is depicted in Figure 4 and 5 respectively. For the first scenario of throughput $0.025 \mathrm{kbps}$ the Queuing delay is $0.003 \mathrm{~ms}$, Medium Access Time is $0.026 \mathrm{~ms}$ and for transmission the time taken is $0.007 \mathrm{~ms}$. The percentage rating of delay and utilization of network is represented in Table 6 and 7.

\subsubsection{Second Scenario}

Figure 6 depicts that the time taken for transmission is minimum among queuing delay and medium access time. As the medium access time is larger, it will results in increase number of retransmission with large collision. The network performance utilization is summarized in Table 8 and 9 respectively.

As in this case, the utilization report is expressed in Figure 7, it shows that the maximum utilization is only $0.010 \%$ with the overhead of $0.010 \%$ and the unutilized

Table 5. Network Statistics- Second Scenario

\begin{tabular}{lr}
\hline \multicolumn{1}{c}{ Metrics } & Value \\
\hline User Level Throughput (kbps) & 7.592 \\
Simulation Time (ms) & 100000 \\
Payload delivered (Bytes) & 894580 \\
Frames Generated & 127268 \\
Frames Transmitted & 60977 \\
Frames Received & 46069 \\
Frames Errored/Collided & 41039 \\
CCA counts & 61350 \\
Successful CCA & 61334 \\
Failed CCA & 15 \\
Routing Overhead (Bytes) & 1238205 \\
\hline
\end{tabular}




\section{Network Performance Delay Report- Second scenario}

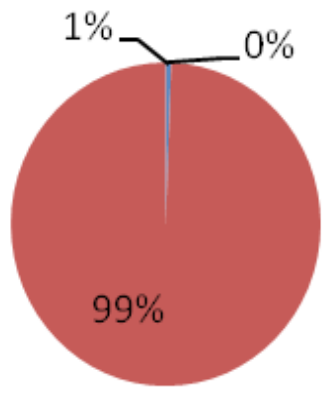

Figure 6. Network Performance Delay Report of First Scenario.

Table 6. Network Delay Percentage

\begin{tabular}{clcc}
\hline $\mathbf{1}$ & \multicolumn{1}{c}{ Delay } & $\mathbf{0 . 0 3 5}(\mathrm{ms})$ & \multicolumn{1}{c}{$\mathbf{1 0 0 \%}$} \\
\hline 1.1 & Queuing Delay & $0.003(\mathrm{~ms})$ & $7.493 \%$ \\
1.2 & Medium Access Time & $0.026(\mathrm{~ms})$ & $73.516 \%$ \\
1.3 & Transmission Time & $0.007(\mathrm{~ms})$ & $18.991 \%$ \\
\hline
\end{tabular}

As in this case the maximum utilization is only $0.010 \%$ with the overhead of $0.010 \%$ and the unutilized network is $99.98 \%$. Because of the low throughput the effective utilization is less than unutilized network.

Table 7. Network Percentage Utilization

\begin{tabular}{clr}
\hline \hline 1 & \multicolumn{1}{c}{ Utilization } & $\mathbf{0 . 0 2 0 \%}$ \\
\hline 1.1 & Effective Utilization & $0.010 \%$ \\
1.2 & Overhead & $0.010 \%$ \\
1.3 & Loss & $0.000 \%$ \\
2 & Unutilized & $99.980 \%$ \\
\hline
\end{tabular}

network is $99.98 \%$. Because of the low throughput the effective utilization is less than unutilized network.

\section{Discussion of Simulation Results}

Here comparative studies of the performance impact of inter node coordination is done by comparing two sets of scenarios.

The simulation results reveal the following

- The synchronization of data from more number of nodes is done effectively by beacon enabled and with acknowledgment request which is more suitable for smart billing system using ZigBee.

- The loss of data and overhead at both the scenarios are very less

- Failed CCA count is very less in both scenarios

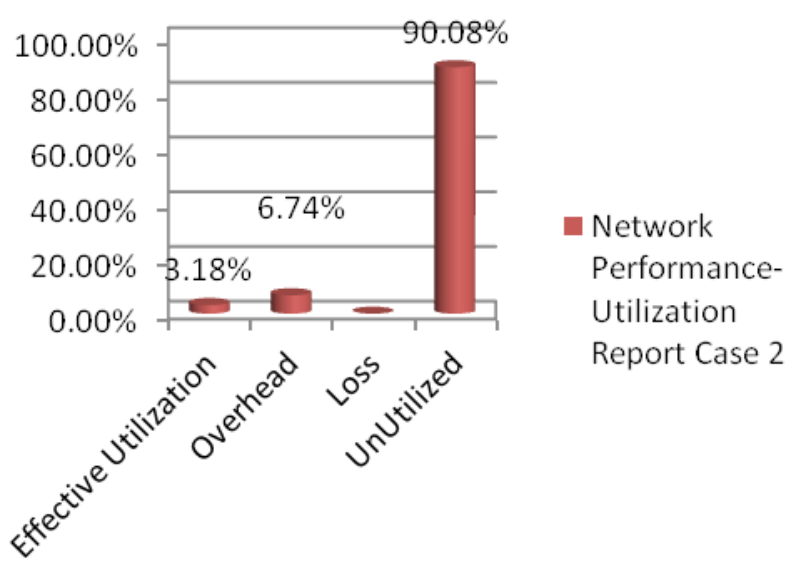

Figure 7. Network Performance Utilization Report of First Scenario.

Table 8. Network Delay Percentage

\begin{tabular}{clrr}
\hline 1 & \multicolumn{1}{c}{ Delay } & $\mathbf{2 1 2 0 9 . 9 4 8}(\mathrm{ms})$ & \multicolumn{1}{c}{$\mathbf{1 0 0 \%}$} \\
\hline 1.1 & Queuing Delay & $120.294(\mathrm{~ms})$ & $0.567 \%$ \\
1.2 & Medium Access Time & $21088.234(\mathrm{~ms})$ & $99.426 \%$ \\
1.3 & Transmission Time & $1.419(\mathrm{~ms})$ & $0.007 \%$ \\
\hline
\end{tabular}

Table 9. Network Percentage Utilization

\begin{tabular}{llr}
\hline \hline 1 & \multicolumn{1}{c}{ Utilization } & $\mathbf{9 . 9 1 9 \%}$ \\
\hline \hline 1.1 & Effective Utilization & $3.181 \%$ \\
1.2 & Overhead & $6.738 \%$ \\
1.3 & Loss & $0.000 \%$ \\
2 & Unutilized & $90.081 \%$ \\
\hline
\end{tabular}

From the simulation the network performance was analyzed. It confirms that ZigBee Cluster Tree Network formation for multiple nodes is well suited for wireless electronic weighing scale for smart billing system.

\section{Conclusion}

The Wireless electronic scale provides an efficient way of weighing goods, update detailed transaction information and exchange of data's and also saves all the information in a host PC. It is less expensive and affordable for an ordinary user. It uses an ultra low power microprocessor with prominent feature makes the scale to operate at low power. Multiple weighing scales in a large supermarket can be connected with a common host PC because of the formation of cluster tree network and synchronization 
of data's is achieved through Beacon enabled mode. The cluster tree network has been formed using nine ZigBee nodes and its network performance was analyzed using NetSim simulator. Thus, this scale provides a sophisticated shopping at vegetable, commercial, retail shops and make convenience in saving the transaction information at low cost.

\section{References}

1. Texas Instruments Incorporated. MSP430FR573x, MSP430FR572x, mixed signal microcontroller. Datasheet SLAS639H; 2013.

2. Lin S, Liu J, Fang Y. ZigBee based wireless sensor networks and its applications in industrial. 2007 IEEE International Conference on Automation and Logistics; 2007 Aug 18-21; Jinan. p. 1979-83.

3. Bensky A. Applications and technologies. In: Short-range wireless communication. 2nd ed. Burlington: Newnes; 2004.

4. Farahani S. ZigBee coexistence. In: ZigBee wireless networks and transceivers. Burlington: Newnes; 2008.

5. Lo Bello L, Toscano E. Coexistence issues of multiple co-located IEEE 802.15.4/ZigBee networks running on adjacent radio channels in industrial environments.
IEEE Transaction on Industrial Informatics. 2009; 5(2): 157-67.

6. Zheng J, Lee M. Will IEEE 802.15.4 make ubiquitous networking a reality: a discussion on a potential low power, low bit rate standard. IEEE Communications Magazine. 2004; 42(6), 140-46.

7. Tetcos Incorporation. NetSim Guide; 2013:110-23.

8. Koubaa A, Cunha A, Alves M, Tovar E. TDBS: a Time division Beacon Scheduling mechanismfor ZigBee clustertree wireless sensor networks. Springer Science+Business Media; 2008.

9. Zhu P, Hu C. Design of wireless electronic scale based on MSP430 microprocessor. AASRI Procedia. 2012; 1:581-87.

10. Sung W-T, Hsu Y-C. Designing an industrial real-time measurement and monitoring system based on embedded system and ZigBee. Expert Systems with Applications. 2011; 38(4):4522-29.

11. Sung W-T, Chen J-H, Chang K-Y. ZigBee based multipurpose electronic score design and implementation using EOG. Sensor Actuator Phys. 2013; 190:141-52.

12. Dannenberg A. MSP430F42x single chip weigh scale. Application Report. Datasheet SLAA220; 2004.

13. Lee J-S, Su Y-W, Shen C-C. Comparative study of wireless protocols: Bluetooth, UWB, ZigBee, and Wi-Fi. IEEE International Symposium on Industrial Electronics Society; 2007 Nov 5-8; Taipei, Taiwan. p. 46-51. 